\title{
Optimal detection bandwidth for phase-sensitive optical time-domain reflectometry
}

\author{
Xin Lu*, Marcelo A. Soto, Luc Thévenaz \\ EPFL Swiss Federal Institute of Technology, Institute of Electrical Engineering, \\ SCI STI LT, Station 11, CH-1015 Lausanne, Switzerland \\ *E-mail: xin.lu@epfl.ch
}

\begin{abstract}
The spectrum of the temporal traces obtained from a phase-sensitive optical time-domain reflectometer is theoretically and experimentally analysed, demonstrating its dependence on the incident optical pulse shape. Numerical simulations and theoretical results are validated experimentally, showing a good matching for rectangular optical pulses. The influence of the photodetector bandwidth on the temporal trace quality is also investigated by simulation and experiment. Results show that the photodetector bandwidth needs to be $\sim 40 \%$ wider than the pulse spectrum to acquire time-domain traces of the Rayleigh backscattered light with direct detection.
\end{abstract}

Keywords: Fibre optics, optical fibre sensors, distributed fibre sensing, Rayleigh scattering,

\section{INTRODUCTION}

Phase-sensitive optical time-domain reflectometry ( $\phi \mathrm{OTDR})$ is a distributed fibre sensing technique that turns attractive for several applications. In a $\phi$ OTDR system, an optical pulse from a coherent light source propagates along a sensing fibre and the random backscattered light interferes within the optical pulse width ${ }^{1}$. Since the size and refractive index of the scattering centres (inhomogeneity of the silica density) giving rise to Rayleigh scattering fluctuate randomly along the fibre, the interference process turns out to be also random, resulting in a time-domain Rayleigh trace with stochastically varying amplitude ${ }^{1}$. The shape of the detected signal is modified by environmental perturbations since both size and refractive index are temperature and strain dependent. Hence, the working principle of the $\phi$ OTDR sensors is based on the changes of the Rayleigh-induced coherent signature measured along the fibre as a result of environmental changes.

Since the temporal shape of the trace varies very fast longitudinally, the detector bandwidth must be large enough to capture this temporal signature; otherwise the stochastically varying trace would be smoothened and the information contained in the trace might be lost $^{2}$. It has been established that the electrical bandwidth required for coherent detection should be at least twice as large as the received signal ${ }^{3}$. However, it is believed that the bandwidth for direct detection should also be several times higher than the pulse spectral bandwidth ${ }^{4}$. Although the spectral feature of the $\phi O T D R$ signal has been investigated, it is still unclear what would be the optical receiver bandwidth to ensure a given spatial resolution in these systems.

In this paper, the temporal and spectral features of $\phi$ OTDR traces obtained by direct detection are analysed by simulation and experiment, when using rectangular optical pulses. The influence of the photodetector bandwidth on the statistical features and visibility of $\phi$ OTDR traces are also investigated. Results demonstrate that a bandwidth $40 \%$ broader than the rectangular pulse intensity spectrum is enough to obtain reliable $\phi$ OTDR traces by direct detection.

\section{THEORY}

An optical fibre can be modelled as a unidimensional medium composed of scattering centres of size $\Delta z$ (being in the order of the optical wavelength) and different refractive index, as shown in Fig. 1. While the light is assumed normally incident on each scattering

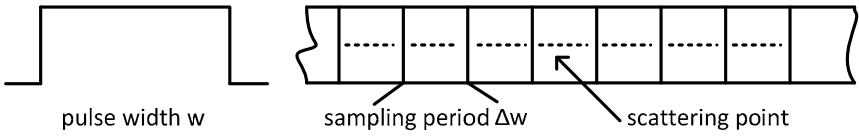

Figure 1. Unidimensional model for coherent Rayleigh scattering. point, the refractive index can be considered as a constant with some longitudinal fluctuations: $n(\mathrm{z})=n_{\text {ave }}+\Delta n(\mathrm{z})$, where 
$n_{\text {ave }}$ is the average refractive index along the fibre and $\Delta n(\mathrm{z})$ is the local index variation. When an optical pulse propagates through the fibre, it is scattered due to the fibre inhomogeneity and the lightwaves reflected within the pulse width $w$ interfere with each other for those reaching the photodetector at the same moment. Thus the field of the Rayleigh backscattered light at position $z$ is written as

$$
\boldsymbol{E}(z)=e^{-\alpha z} \sum_{m=0}^{M} P_{E}(m) \cdot \boldsymbol{E}_{\boldsymbol{0}} \boldsymbol{r}(z+m \cdot \Delta z) e^{j \cdot 2 \phi(z+m \cdot \Delta z)}
$$

where $\alpha$ is the fibre attenuation coefficient, $M=w / \Delta z$ represents the number of scattering points inside the pulse, $r(z)$ is the complex reflection coefficient, $\boldsymbol{E}_{0}$ is the peak of the optical pulse field, $P_{\mathrm{E}}$ is the normalized pulse shape and the optical phase $\phi(z+m \Delta z)$ is the integration of the propagation constant $\beta(z)=2 \pi n(z) v / \mathrm{c}$ from $z$ to $z+m \Delta z$, where $c$ is the light speed in vacuum and $v$ is the optical frequency. Equation (1) shows that coherent Rayleigh scattering is essentially a random walk process. This means that the amplitude of the field is a Rayleigh-distributed random variable, while the normalised intensity follows an exponentially-decaying distribution ${ }^{1,4}$.

The spatial resolution of a $\phi O T D R$ system (given by the pulse width) is typically a few metres, so that the fibre loss within the pulse width can be safely neglected. In addition, the factor $M$ in Eq. (1) is usually large enough to approximate the average refractive index by $n_{\text {ave }}$ within the interval from $z$ to $z+(m-1) \Delta z$. Thus the optical phase can be written as:

$$
\phi(z+m \cdot \Delta z)=\int_{z}^{z+m \cdot \Delta z} \frac{2 \pi n(\xi) v}{c} d \xi=\frac{2 \pi v}{c} \sum_{i=0}^{m} n(z+i \cdot \Delta z) \cdot i \Delta z \approx \frac{2 \pi v \Delta z}{c}\left[m \cdot n_{\text {ave }}+\Delta n(z+m \cdot \Delta z)\right] .
$$

If the refractive variation along the fibre is given, the backscattered light in the $\phi$ OTDR system can be calculated based on Eqs. (1) and (2).

The spectral characteristics have been studied based on Eq. (1) and the power spectrum density (PSD) of the reflected light amplitude $E$ and intensity $I$ can be respectively expressed as ${ }^{5,6}$ :

$$
\begin{gathered}
S_{E}(f)=\left\langle H_{E} H_{E}^{*}\right\rangle S_{E}^{P}=I_{0} r_{0}^{2} \times S_{E}^{P}=\left.I_{0} r_{0}^{2} \nmid \mathrm{F}\left\{P_{E}\right\}\right|^{2}, \\
S_{I}(f)=I_{0}^{2} r_{0}^{4} \delta(f)+S_{E}(f) \otimes S_{E}(f),
\end{gathered}
$$

where * denotes the conjugation, $<>$ represents ensemble average, $I_{0}$ is the optical intensity, $r_{0}{ }^{2}=<r r^{*}>, S_{\mathrm{E}}{ }^{\mathrm{P}}$ is the PSD of the pulse, $\delta$ represents the delta function and $\mathrm{F}\{\}$ denotes Fourier transform. Equation (3) shows that the spectrum of the backscattered light amplitude $S_{\mathrm{E}}$ keeps the same shape as the optical pulse intensity spectrum, which is a sinc-square shape for rectangular pulse. And the $3 \mathrm{~dB}$ bandwidth of the intensity spectrum for the rectangular pulse is calculated to be $0.44 / T$, where $T$ is the pulse width in the time domain. In the case of direct detection, all the frequency components of the backscattered light field beat with each other on the photodetector, represented by the second term on the right hand side of Eq. (4), resulting in a broader spectrum for a rectangular input pulse. Therefore, it is reasonable to expect that a photodetector with a bandwidth larger than the pulse spectrum width may be required.

In the next section, the electrical spectrum of the measured time-domain Rayleigh traces obtained by numerical simulations of Eqs. (1)-(2) are compared with the one obtained from Eqs. (3) and (4), for the rectangular pulse. Results are also validated by experimental measurements using a $\phi$ OTDR system employing direct detection.

\section{SIMULATION AND EXPERIMENT}

To validate the theory and mathematical models presented in the last section, a coherent Rayleigh trace originating from a $1 \mathrm{~km}$ single mode fibre is measured by a standard $\phi$ OTDR system based on direct detection. Rectangular pulses of temporal width $T=10 \mathrm{~ns}$ are employed to validate the theory and simulation results. Part of the backscattered light enters a high resolution optical spectrum analyser (OSA) operating in coherent detection mode, so that the power spectral density of optical amplitude $S_{\mathrm{E}}$ is shown by the OSA. The other part is obtained by a photodetector with $1 \mathrm{GHz}$ bandwidth, which is large enough compared to the spectral FWHM of the rectangular pulses, to avoid unexpected distortions in the time-domain traces. Temporal Rayleigh traces are acquired by an oscilloscope with $4 \mathrm{GHz}$ bandwidth, while the corresponding power spectral density of these traces intensity is measured using an electric spectrum analyser. 
The field amplitude of the Rayleigh scattering can be simulated by Eqs. (1)-(2) using a randomly generated refractive index $\Delta n(z)$, while the reflection coefficient can be calculated based on the Fresnel equation. However this method is very inefficient and time-consuming because there exist millions of scattering points contributing to the Rayleigh scattering interfering inside the pulse width. In addition, an exhaustive simulation considering each scattering point is unnecessary since the time-domain Rayleigh trace is sampled by the acquisition system at discrete points with a sampling interval $\Delta w$, so that each data point contain the interference resulting from the Rayleigh light backscattered from thousands scattering points. The workload reduces greatly by substituting the index $\Delta n(z)$ in Eqs. (1)-(2) by an equivalent factor $\Delta n^{\prime}\left(z_{k}\right)$ at each sampled location $z_{k}$. Since the number of scattering points inside the sampling interval is still large enough, the index variation is assumed to follow a Gaussian distribution as justified by the central limit theorem ${ }^{1}$. Thus, while the average refractive index $n_{\text {ave }}$ is considered equal to 1.46 , the refractive index variation $\Delta n^{\prime}\left(z_{k}\right)$ is randomly generated at each sampling point $z_{k}$ based on a zero-mean Gaussian probability distribution with a standard deviation of $2 \times 10^{-6}$. Equations (1)-(2) are solved numerically considering a $1 \mathrm{~km}$-long single-mode fibre, neglecting the fibre loss and using $10 \mathrm{~ns}$ rectangular pulses. Numerical results are simulated with an equivalent sampling rate of $1 \mathrm{GS} / \mathrm{s}$ to match the oscilloscope setting used in the experiment. Finally the intensity PSD as delivered by the electrical spectrum analyser is obtained by calculating the Fourier transform of the simulated time-domain Rayleigh traces.

According to Eq. (3), the spectral shape of the Rayleigh backscattered light amplitude should be sinc-squared for the rectangular pulse. The spectral width of the backscattered light intensity can also be obtained from Eq. (4) with the corresponding pulse shape.

The spectra of backscattered light amplitude and intensity are plotted in Fig. 2, for rectangular pulse. Simulation and experimental results agree well with the theoretical prediction given by Eqs. (3) and (4). Figure 2(a) shows that the $3 \mathrm{~dB}$ width of the amplitude PSD is $\sim 44.3 \mathrm{MHz}$, being identical to the spectral width of the used rectangular pulses. On the other hand, the inset of Fig. 2(b) shows the 3-dB width of the intensity spectrum $f_{3 \mathrm{~dB}}$ is $59 \mathrm{MHz}$, which is larger than the bandwidth needed to detect the corresponding rectangular pulse. Simulation results with different pulse width reveal the relationship $f_{3 \mathrm{~dB}}=0.6 / T$, the same as the reported result.
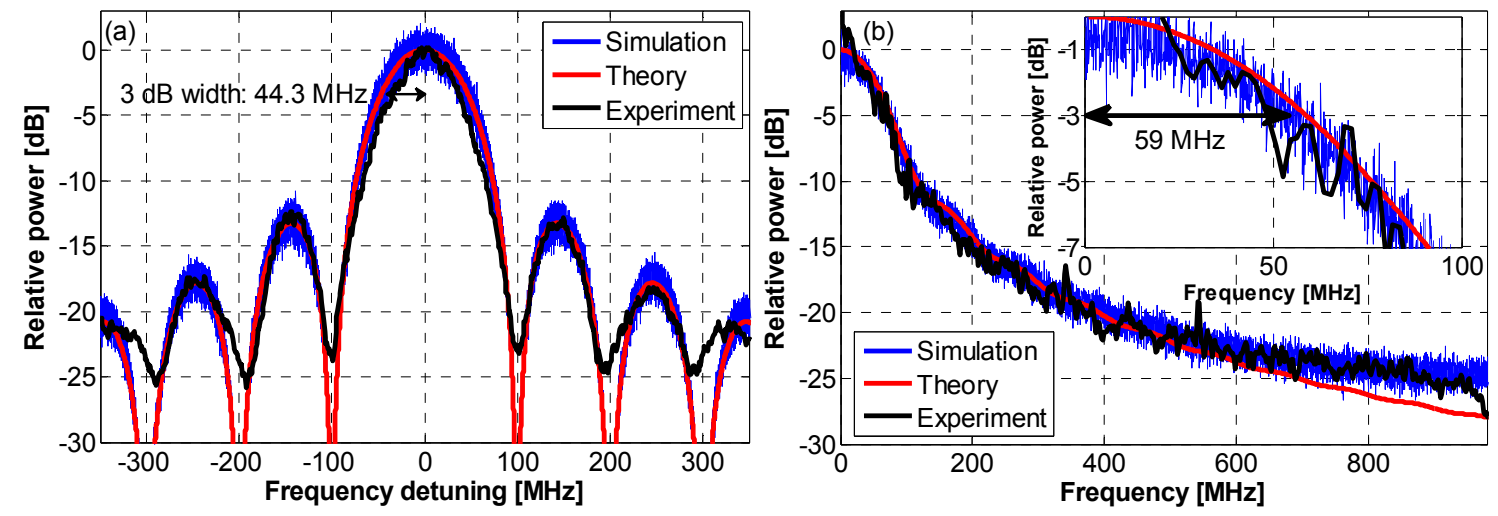

Figure 2. Spectra of the $\phi$ OTDR trace with rectangular input pulse obtained by (a) an optical spectrum analyser (amplitude PSD), and

(b) direct detection and electrical spectrum analyser (intensity PSD), inset corresponds to a zoom of the low frequency range.

Finally the impact of the photodetector bandwidth on the time-domain trace is investigated for the rectangular pulse case. In the experiment, different bandwidths are flexibly obtained by changing the internal digital filter of the oscilloscope. The limited bandwidth smooths the temporal trace, thus changing its statistical features. Figure 3(a) shows the probability density of the traces normalised to its mean value for different filter bandwidths. Since the coherent Rayleigh scattering can be identified to a random walk process, the probability density of the obtained intensity traces obeys to an exponentially decaying distribution. Figure 3(a) demonstrates that simulation and experimental results show the same tendency when the bandwidth is sufficiently large; however the when the electrical bandwidth is reduced, the maximum of the probability density shifts to the mean value of the trace (here represented as 1 due to the normalisation) and becomes narrower and higher in amplitude. This is because the random Rayleigh trace is smoothed and turns into a more flattened trace. This bandwidth reduction is actually detrimental to the trace quality, typically quantified by the visibility ${ }^{2}$ $V=\left(I_{\max }-I_{\min }\right) /\left(I_{\max }+I_{\min }\right)$. As presented in Fig. 3(b), the visibility remains high when the electrical bandwidth is reduced from $1 \mathrm{GHz}$ down to $125 \mathrm{MHz}$. However, the visibility drops dramatically for bandwidths below $125 \mathrm{MHz}$, impairing the quality of the traces and the subsequent extraction of information. 

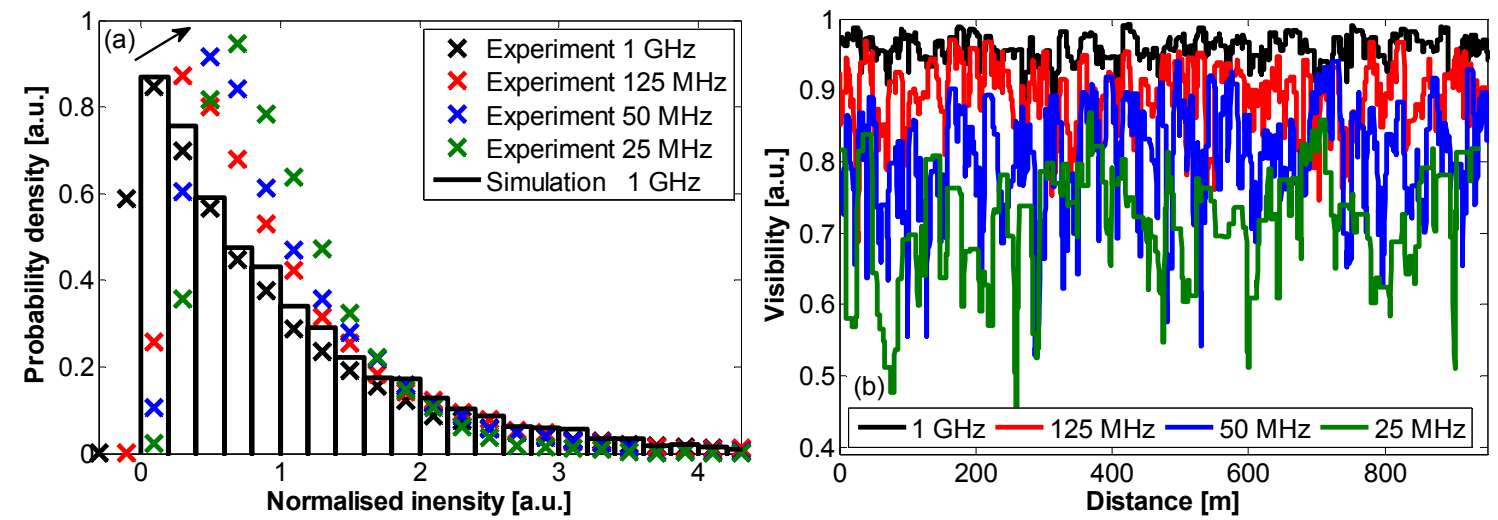

Figure 3. Influence of the photodetector bandwidth on a. statistical distribution of intensity and b. visibility of the $\phi O T D R$ intensity trace.

The analysis of the Rayleigh backscattered light in time and frequency domain demonstrates that $\sim 60 \mathrm{MHz}$ is the minimum requirement for the detector $3 \mathrm{~dB}$ bandwidth to obtain a comparatively high quality $\phi O T D R$ signal using $10 \mathrm{~ns}$ rectangular pulse. Based on the simulation of different pulse widths, a minimum bandwidth of $0.6 / T$ is found to acquire the OTDR intensity trace with good quality. As a result, the analysis of the Rayleigh backscattered light in time and frequency domain demonstrates that the photo receiver bandwidth to obtain the $\phi$ OTDR by direct detection should be at least $(0.6 / T) /(0.44 / T) \approx 1.36$ times as broad as the bandwidth to detect the incident rectangular pulse.

\section{CONCLUSION}

From a theoretical model the temporal and spectral properties of the $\phi$ OTDR signal is analysed and the optimal bandwidth to acquire the Rayleigh intensity trace is determined and experimentally validated. Results confirm that a receiver bandwidth $\sim 40 \%$ broader than the rectangular pulse spectrum is enough for the $\phi$ OTDR system with direct detection. According to several studies rectangular pulses are believed to be the best shape for time-domain distributed sensing, but this result may substantially differ for other pulse shapes and the bandwidth requirement can be investigated based on the proposed model.

\section{REFERENCES}

[1] Zhou, J., Pan, Z., Ye, Q., Cai, H., Qu, R. and Fang, Z., "Characteristics and explanations of interference fading of a phi-OTDR with a multi-frequency source," J. Lightw. Technol., 31(17), 2947-2954 (2013).

[2] Staubli, R. K., and Gysel, P., "Statistical properties of single-mode fiber Rayleigh backscattered intensity and resulting detector current," IEEE Trans. Commun., 40(6), 1091-1097 (1992).

[3] Ip, E., et al.,"Coherent detection in optical fiber systems," Opt. Express, 16(2), 753-791 (2008).

[4] Martins, H. F., et al., "Coherent noise reduction in high visibility phase-sensitive optical time domain reflectometer for distributed sensing of ultrasonic waves," J. Lightw. Technol., 31(23), 3631-3637 (2013).

[5] Gysel, P., and Staubli, R. K., "Spectral properties of Rayleigh backscattered light from single-mode fibres caused by a modulated probe signal," J. Lightw. Technol., 8(12), 1792-1798 (1990).

[6] Alekseev, A. E., Vdovenko, V. S., Gorshkov, B. G., Potapov, V. T. and Simikin, D. E., "A phase-sensitive optical time-domain reflectometer with dual-pulse diverse frequency probe signal," Laser Phys., 25(6), 065101 (2015). 